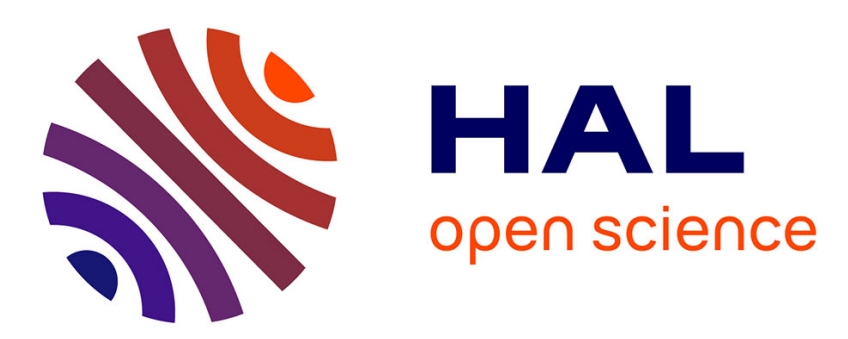

\title{
A diagonalization algorithm revisited and applied to the nuclear shell model
}

D Bianco, F Andreozzi, N Lo Iudice, A Porrino, F Knapp

\section{To cite this version:}

D Bianco, F Andreozzi, N Lo Iudice, A Porrino, F Knapp. A diagonalization algorithm revisited and applied to the nuclear shell model. Journal of Physics G: Nuclear and Particle Physics, 2011, 38 (2), pp.25103. 10.1088/0954-3899/38/2/025103 . hal-00600877

\section{HAL Id: hal-00600877 \\ https://hal.science/hal-00600877}

Submitted on 16 Jun 2011

HAL is a multi-disciplinary open access archive for the deposit and dissemination of scientific research documents, whether they are published or not. The documents may come from teaching and research institutions in France or abroad, or from public or private research centers.
L'archive ouverte pluridisciplinaire HAL, est destinée au dépôt et à la diffusion de documents scientifiques de niveau recherche, publiés ou non, émanant des établissements d'enseignement et de recherche français ou étrangers, des laboratoires publics ou privés. 


\title{
A diagonalization algorithm revisited and applied to nuclear shell model
}

\author{
Bianco $\mathbf{D}^{1}$, Andreozzi $\mathbf{F}^{1}$, Lo Iudice $\mathbf{N}^{1}$, Porrino $\mathbf{A}^{1}$, Knapp $\mathbf{F}^{2} \ddagger$ \\ ${ }^{1}$ Dipartimento di Scienze Fisiche, Università di Napoli Federico II, and INFN, \\ Sezione di Napoli, Napoli, Italy \\ ${ }^{2}$ INFN, Sezione di Napoli, Napoli, Italy \\ E-mail: loiudice@na.infn.it
}

\begin{abstract}
An importance sampling iterative algorithm for diagonalizing large matrices is upgraded and adopted for large scale nuclear shell model calculations using a spin uncoupled basis. Its numerical implementation shows that the iterative procedure converges rapidly to the exact eigensolutions achieving an effective drastic cut of the sizes of the Hamiltonian matrix.
\end{abstract}

PACS numbers: 21.60.Cs,23.20.Lv

$\ddagger$ Present address: Faculty of Mathematics and Physics, Charles University, Prague, Czech Republic 


\section{Introduction}

Lanczos algorithm [1, 2] is widely adopted in several branches of physics and other disciplines for diagonalizing large size matrices. Its many implementations in nuclear structure are devoted to the shell model (SM) eigenvalue problem.

Among them, some codes adopt a basis of states projected in spin (or spinisospin) $(j-j$-scheme) $[3,4,5,6]$ so as to deal with Hamiltonian matrices of relatively small dimensions. These matrices, however, are very dense and require lengthy and cumbersome calculations of fractional parentage and recoupling coefficients.

Other adopt the unprojected $m$-scheme. In such a basis, the Hamiltonian matrix, though of much larger dimensions, has a far simpler structure and is sparse. Both properties are fully exploited in the powerful Antoine code [3, 4].

Alternative to Lanczos is the shell model Monte Carlo (SMMC) [7], which avoids the diagonalization of the Hamiltonian matrix by adopting a Monte Carlo technique to evaluate expectation values and strength functions. The SMMC, however, is not suited for computing spectra and has to face the sign problem which has not found a clear-cut solution yet.

The MC technique is used in the quantum Monte Carlo diagonalization (QMCD) method [8, 9] just to generate stochastically a truncated basis. This is then used to diagonalize the Hamiltonian. Specific projection methods had to be developed in order to restore the angular momentum and other symmetries, broken by the stochastic sampling. The QMCD was adopted for systematics, especially in the $(p, f)$ region.

Another technique for truncating the basis is provided by the density matrix renormalization group (DMRG) [10], borrowed from condensed matter [11], based on a partitioning of the full space into smaller subspaces. This method seems to be well suited to schematic Hamiltonians. When applied to shell model, it seems to work better in the coupled scheme [12].

Coming back to Lanczos, its numerical implementation deserves special care in order to avoid numerical errors, whose propagation, during the iterative process, may yield "ghost" states.

Few years ago $[13,14]$, we proposed an iterative algorithm, alternative to Lanczos, which is free of instabilities and of easy implementation. It is also endowed with an importance sampling, which achieves a truncation of the basis. The algorithm was implemented in the coupled $j$-scheme only, which is too time consuming in the iterative construction of the Hamiltonian matrix. Here, we adopt the $m$-scheme and elaborate an efficient variant of the method which promises to be considerably more competitive. Its performance will be illustrated through some numerical examples.

The algorithm is as general as Lanczos and, therefore, is not bound to nuclear structure. It may be used in other fields of physics and outside of the realm of physics. 


\section{The algorithm}

The algorithm has been described in Refs. $[13,14]$. We give here a brief outline. Let us consider a matrix $A=\left\{a_{i j}\right\}$ representing a self-adjoint operator $\hat{A}$ in an orthonormal basis $\{|1\rangle,|2\rangle, \ldots,|i>, \ldots| N\rangle$,$\} . The algorithm consists of several iteration loops.$ The first loop goes through the following steps :

a) Pick up the lowest $n_{0}$ basis states $\mid i>\left(n_{0}<<N\right)$, construct and diagonalize the $n_{0} \times n_{0}$ submatrix $A_{0}=\left(a_{i j}\right)$,

b)select the lowest $v$ eigenvalues $\Lambda_{0} \equiv\left\{\lambda_{1}^{(0)}, \ldots, \lambda_{k}^{(0)}, \ldots, \lambda_{v}^{(0)}\right\}$ and the corresponding eigenvectors

$$
\left|\varphi_{k}^{(0)}\right\rangle=\sum_{i=1}^{n_{0}} c_{i}^{(0)}|i\rangle
$$

c) consider the subspace spanned by these $v$ eigenvectors $\left|\varphi_{k}^{(0)}\right\rangle$ plus the basis states $|j>=| n_{0}+1>, \ldots \mid n_{1}>$ and construct the new submatrix

$$
A_{1}=\left(\begin{array}{cc}
\Lambda_{0} & B_{01} \\
B_{10} & C_{1}
\end{array}\right)
$$

composed of the diagonal block $\Lambda_{0} \equiv\left\{\lambda_{k}^{(0)}\right\}$ plus $C_{1}=\left\{a_{i j}\right\}\left(i, j=n_{0}+1, n_{1}\right)$. The two blocks are coupled by $B_{01}$ and its transpose, composed of the updated matrix elements $b_{k j}^{(0)}=\left\langle\varphi_{k}^{(0)}|\hat{A}| j\right\rangle(k=1, v),\left(j=n_{0}+1, n_{1}\right)$,

d) diagonalize $A_{1}$, extract the new lowest $v$ eigenvalues $\Lambda_{1} \equiv\left\{\lambda_{k}^{(1)}\right\} \quad(k=1, v)$ and eigenvectors $\left|\varphi_{k}^{(1)}\right\rangle$, and use them plus the basis states $\mid j>\left(j=n_{1}+1, n_{2}\right)$ to construct a new submatrix $A_{2}$ just as done in c).

The iterative procedure is now clearly outlined and can be illustrated by the following sequential operations

$$
\begin{aligned}
A_{0} \Longrightarrow \Lambda_{0} \longrightarrow & A_{1}=\left(\begin{array}{cc}
\Lambda_{0} & B_{01} \\
B_{10} & C_{1}
\end{array}\right) \Longrightarrow \Lambda_{1} \longrightarrow \ldots \\
& \longrightarrow A_{n}=\left(\begin{array}{cc}
\Lambda_{(n-1)} & B_{(n-1) n} \\
B_{n(n-1)} & C_{n}
\end{array}\right) \Longrightarrow \Lambda_{n}=\Lambda .
\end{aligned}
$$

The first iteration loop goes on until the basis is exhausted, yielding the approximate $v$ eigenvalues and eigenvectors

$$
E_{k}^{(1)} \equiv \lambda_{k}^{(n)}, \quad\left|\psi_{k}^{(1)}\right\rangle \equiv\left|\varphi_{k}^{(n)}\right\rangle=\sum_{i=1}^{N} c_{k}^{(n)}(i)|i\rangle .
$$

These $v$ eigenvectors $\mid \psi_{k}^{(1)}>$ added to the original basis states $\{|i\rangle\}$ are the entry for a new iteration loop. Since these states form a non orthogonal redundant basis, we solve a generalized eigenvalue problem and adopt the Choleski decomposition method to eliminate any redundancy. Apart from this modification, the subsequent iteration loops proceed as the first one and generate a sequence converging to the exact eigensolutions $[13]$

$$
\left\{E_{k}^{(r)}, \psi_{k}^{(r)}\right\} \stackrel{r \rightarrow \infty}{\longrightarrow}\left\{E_{k}, \psi_{k}\right\}
$$


As proved and illustrated through typical numerical tests [13], the algorithm is robust, being numerically stable and converging always to the extremal eigenvalues. It yields ghost-free solutions and is also of easy implementation.

It requires $\mathrm{O}\left(N^{2}\right)$ operations. In fact we need $N$ operations to compute the updated

matrix element $b_{k j}^{(n-1)}=\left\langle\lambda_{k}^{(n-1)}|\hat{A}| j\right\rangle(k=1, v)$. For sparse matrices with an average number $L$ of non zero matrix elements, the required number of operations grows linearly with $N$.

\section{Implementation of the algorithm in the $m$-scheme}

Let $\hat{A}$ be a SM Hamiltonian $H$ of a nucleus with $p$ valence nucleons. These are supposed to be described by the basis states $\left.|i>=| \alpha_{1}, \ldots \alpha_{i}, \ldots, \alpha_{p}\right\rangle$, where $\alpha_{i}=a_{i} m_{i}$ are the quantum numbers of a particle in the shell $a_{i}=\left\{n_{i} l_{i} j_{i}\right\}$. The states $\mid i>$ have the total magnetic quantum number $M=m_{1}+\ldots m_{i}+\ldots m_{p}$.

It is advantageous to adopt the modified Hamiltonian

$$
H_{J}=H+c\left[\mathbf{J}^{2}-J(J+1)\right]^{2},
$$

where $\mathbf{J}$ is the total spin operator, $J$ a given integer (or half-integer), and $c$ a positive constant.

The full space can be thought to be decomposed into several subspaces

$$
\mathcal{H}=\mathcal{H}_{0} \oplus \mathcal{H}_{1} \ldots \oplus \mathcal{H}_{k} \ldots \oplus \mathcal{H}_{F}
$$

Each subspace $\mathcal{H}_{k}$ is composed of a set of partitions $\left\{n_{i}\right\}_{k}=\left\{a_{1}^{n_{1}}, \ldots a_{i}^{n_{i}}, \ldots\right\}_{k}$, where $\sum_{i} n_{i}=p$. It is, therefore, invariant with respect to $\mathbf{J}$. The partitions in $\mathcal{H}_{k}$ differ from those in $\mathcal{H}_{k-1}$ by at most two single particle shells $a_{i}$.

We proceed according to the following iterative scheme

$$
\mathcal{H}_{0} \Rightarrow \Lambda_{0} \longrightarrow \Lambda_{0} \oplus \mathcal{H}_{1} \Rightarrow \Lambda_{1} \longrightarrow \ldots \longrightarrow \Lambda_{F-1} \oplus \mathcal{H}_{F} \Rightarrow \Lambda_{F} .
$$

We, namely, diagonalize the Hamiltonian $H_{J}$ in $\mathcal{H}_{0}$ obtaining $v$ lowest eigenvalues $E_{1}^{(0)}, \ldots, E_{v}^{(0)}$ and eigenvectors $\psi_{1}^{(0)}, \ldots, \psi_{v}^{(0)}$ spanning a subspace $\Lambda_{0}$. These eigensolutions are exact in this subspace and have all the same spin $J$ if the constant $c$ is chosen so as to push the states with $J^{\prime} \neq J$ up in energy.

Because of its two-body nature, the Hamiltonian couples the subspace $\Lambda_{0}$ to $\mathcal{H}_{1}$ only. We therefore diagonalize $H_{J}$ in the subspace $\Lambda_{0} \oplus \mathcal{H}_{1}$ to generate new updated eigenvalues $E_{1}^{(1)}, \ldots, E_{v}^{(1)}$ and eigenvectors $\psi_{1}^{(1)}, \ldots, \psi_{v}^{(1)}$, defining the subspace $\Lambda_{1}$. We proceed iteratively. Once the updated eigensolutions defining the subspace $\Lambda_{k}$ are obtained, we diagonalize the Hamiltonian in the upgraded subspace $\Lambda_{k} \oplus \mathcal{H}_{k+1}$. We cover eventually the full space obtaining the exact $v$ eigensolutions $\left\{E_{i}, \psi_{i}\right\}$.

By exploiting the sparsity of the Hamiltonian matrix, the above procedure allows to reduce the number of operations to $O(N)$, as in Lanczos implementations. This, however, may not be sufficient for spaces of very large dimensions. It is therefore useful to search for a reliable way of cutting the basis. To this purpose, the original sampling procedure [14] has been modified and adapted to the $m$-scheme. 
Table 1. Single proton particle and single neutron hole energies (in MeV).

\begin{tabular}{llllll}
\hline$(n l j)_{p}$ & $2 d_{5 / 2}$ & $1 g_{7 / 2}$ & $2 d_{3 / 2}$ & $3 s 1 / 2$ & $1 h_{11 / 2}$ \\
\hline$\epsilon_{p}$ & 0.00 & 0.2 & 2.2 & 2.3 & 2.9 \\
\hline$(n l j)_{n}^{-1}$ & $\left(2 d_{3 / 2}\right)^{-1}$ & $\left(1 h_{11 / 2}\right)^{-1}$ & $\left(3 s_{1 / 2}\right)^{-1}$ & $\left(2 d_{5 / 2}\right)^{-1}$ & $\left(1 g_{7 / 2}\right)^{-1}$ \\
\hline$\epsilon_{n}$ & 0.00 & 0.14 & 0.33 & 1.65 & 2.43 \\
\hline
\end{tabular}

Let us fix a sequence of positive small numbers of decreasing values $\epsilon_{1}>\ldots \epsilon_{k}>$ $\ldots>\epsilon_{F}$. Having generated the lowest $v$ eigenvectors $\psi_{1}^{(0)}, \ldots, \psi_{v}^{(0)}$ in $\mathcal{H}_{0}$, we proceed following the same scheme (8) of the exact case with one constraint: In going from $\mathcal{H}_{k-1}$ to $\mathcal{H}_{k}$, we pick up only the basis states $\mid j>$ that fulfill the condition

$$
\frac{|<j| H_{J}\left|\varphi_{i}^{(k-1)}>\right|^{2}}{a_{j j}-E_{i}^{(k-1)}}>\epsilon_{k} .
$$

More specifically, in the first step $(k=1)$, the above condition selects a set of states $\{\mid j>\}$ forming a subspace $\mathcal{H}_{1}^{\left(\epsilon_{1}\right)} \in \mathcal{H}_{1}$. The eigenvalue problem is thus solved in $\Lambda_{0} \oplus \mathcal{H}_{1}\left(\epsilon_{1}\right)$ yielding new $v$ eigensolutions $E_{k}^{(1)}\left(\epsilon_{1}\right), \psi_{k}^{(1)}\left(\epsilon_{1}\right)$ defining the subspace $\Lambda_{1}^{\left(\epsilon_{1}\right)}$. We now explore the full subspace complementary to $\mathcal{H}_{0} \oplus \mathcal{H}_{1}\left(\epsilon_{1}\right)$ and select all the states $\mid j>$ that fulfill the condition (9) with $\epsilon_{2}$ replacing $\epsilon_{1}$. The states so selected span a subspace $\mathcal{H}_{2}^{\left(\epsilon_{2}\right)} \in \mathcal{H}_{0} \oplus \mathcal{H}_{1} \oplus \mathcal{H}_{2}$. The above procedure is iterated with updated eigensolutions and decreasing sampling values $\epsilon_{k}$ until the full space is covered.

A sketch of the sampling procedure is provided by the following sequence

$$
\mathcal{H}_{0} \Rightarrow \Lambda_{0} \stackrel{\epsilon_{1}}{\longrightarrow} \Lambda_{0} \oplus \mathcal{H}_{1}^{\left(\epsilon_{1}\right)} \Rightarrow \Lambda_{1}^{\left(\epsilon_{1}\right)} \stackrel{\epsilon_{2}}{\longrightarrow} \ldots \stackrel{\epsilon_{F}}{\longrightarrow} \Lambda_{F-1}^{\left(\epsilon_{F-1}\right)} \oplus \mathcal{H}_{F}^{\left(\epsilon_{F}\right)} \Rightarrow \Lambda_{F}^{\left(\epsilon_{F}\right)} \text {.(10) }
$$

Clearly, we get the exact solutions for $\epsilon_{F} \rightarrow 0$, namely $\lim _{\epsilon_{F} \rightarrow 0} \Lambda_{F}^{\left(\epsilon_{F}\right)}=\Lambda_{F}$, or, more explicitly,

$$
\lim _{\epsilon_{F} \rightarrow 0}\left\{E_{i}\left(\epsilon_{F}\right), \psi_{i}\left(\epsilon_{F}\right)\right\}=\left\{E_{i}, \psi_{i}\right\} .
$$

Few remarks are mandatory. Like the algorithm sketched in Section 2, the sampling procedure is extremely stable. It yields, indeed, orthonormal eigenfunctions. These, at each step, result from the diagonalization of a symmetric submatrix. The subspaces selected by the sampling are not strictly invariant with respect to $\mathbf{J}$. The invariance, however, is restored as the sampling value $\epsilon$ becomes sufficiently small. We will show, in fact, that all sampled eigenstates reach soon a good $J$.

The just outlined sampling process reminds of the DMRG [10]. In both approaches, in fact, one starts with a subspace of small dimensions $n_{0}$ and then goes through several steps consisting of enlarging the space and, then, going back to a restricted space of the original dimensions $n_{0}$ spanned by updated eigensolutions. There are crucial differences, however. Ours is based on a new diagonalization algorithm. Moreover, in our case the space truncation is achieved by updating both energies and wavefunctions, while in the DMRG it is based on prescriptions imposed on the wave functions (density matrix). 


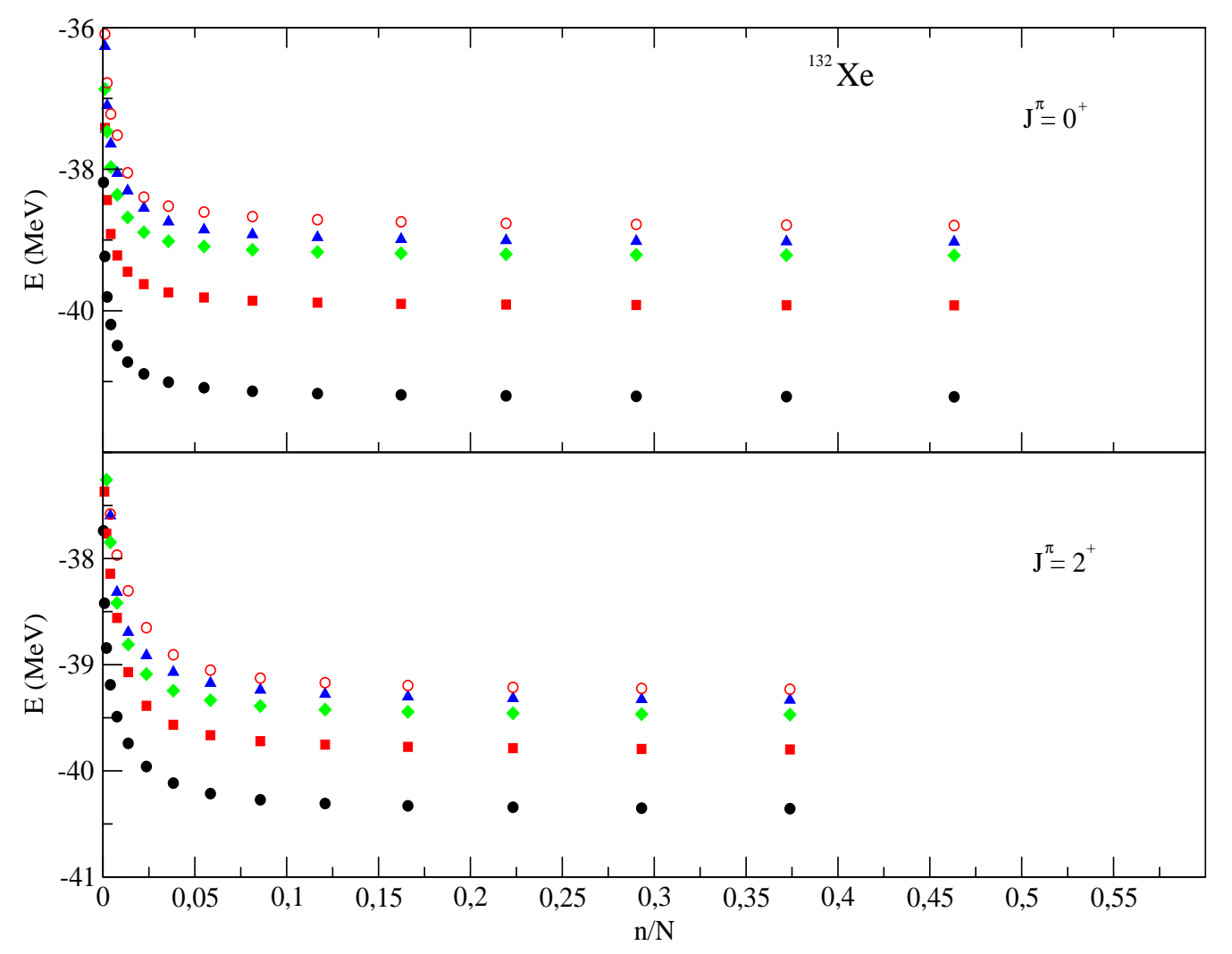

Figure 1. Convergence rate of the lowest $J^{\pi}=0^{+}$and $J^{\pi}=2^{+}$eigenvalues in ${ }^{132} \mathrm{Xe}$.

\section{Numerical implementation and results}

We applied the sampling algorithm to ${ }^{132} \mathrm{Xe}$ and ${ }^{130} \mathrm{Xe}$. These nuclei are supposed to be near the transition point from a vibrational to a $\gamma$-soft structure and are being object of experimental investigations [15].

The model space includes the shells $\{2 d 5 / 2,1 g 7 / 2,2 d 3 / 2,3 s 1 / 2,1 h 11 / 2\}$ for both valence neutrons and protons. The dimensions of the resulting Hamiltonian matrix are $N \simeq 3.7 \times 10^{7}$ for ${ }^{132} \mathrm{Xe}$ and $N \simeq 0.8 \times 10^{9}$ for ${ }^{130} \mathrm{Xe}$.

It is advantageous to describe the above isotopes in terms of valence proton particles external to a $Z=50$ core and neutron holes referredl to a $N=82$ core. The single particle energies are shown in Table 1 . The neutron hole energies were deduced from the spectrum of ${ }^{131} \mathrm{Sn}$, while the proton particle energies were taken from Ref. [14].

A renormalized G matrix [16] derived from the CD-Bonn potential [17] was taken as a two-body interaction. The $E 2$ transitions were computed using the effective charges $e_{p}=1.5$ and $e_{n}=0.7$ for protons and neutrons, respectively.

The first task consisted in choosing the initial subspace $\mathcal{H}_{0}$. Its dimensions $n_{0}$ increase with the number of eigenstates of good $J$ we intend to generate. To yield up to ten eigenstates, the space dimensions came out to be of the order $n_{0} \sim 100$. Having chosen $\mathcal{H}_{0}$, we applied the iterative sampling procedure (10) with decreasing values of $\epsilon_{k}$. Each $\epsilon_{k}$ determines uniquely the dimension $n_{k}$ of the Hamiltonian matrix to be 
Table 2. Convergence rate of the absolute energies (in $\mathrm{MeV}$ ) in ${ }^{130} \mathrm{Xe}$ and ${ }^{132} \mathrm{Xe}$.

\begin{tabular}{|c|c|c|c|c|c|c|c|c|}
\hline \multirow[t]{2}{*}{${ }^{132} \mathrm{Xe}$} & \multirow[t]{2}{*}{$J^{\pi}=0^{+}$} & \multirow{2}{*}{\multicolumn{2}{|c|}{$J_{\text {samp }}$}} & \multicolumn{5}{|c|}{$(n / N)_{k}$} \\
\hline & & & & 0.081 & 0.219 & 0.290 & 0.372 & 0.463 \\
\hline & $0_{1}^{+}$ & 0.0005 & $E_{k}=$ & -41.138 & -41.201 & -41.209 & -41.213 & -41.215 \\
\hline & $0_{2}^{+}$ & 0.0004 & $E_{k}=$ & -39.857 & -39.912 & -39.918 & -39.922 & -39.924 \\
\hline & \multirow[t]{2}{*}{$J^{\pi}=2^{+}$} & \multirow{2}{*}{\multicolumn{2}{|c|}{$J_{\text {samp }}$}} & & & \multicolumn{3}{|l|}{$(n / N)_{k}$} \\
\hline & & & & 0.086 & 0.166 & 0.223 & 0.293 & 0.374 \\
\hline & $2_{1}^{+}$ & 2.0002 & $E_{k}=$ & -40.273 & -40.329 & -40.343 & -40.351 & -40.356 \\
\hline & $2_{2}^{+}$ & 2.0001 & $E_{k}=$ & -39.720 & -39.774 & -39.786 & -39.794 & -39.799 \\
\hline \multirow[t]{8}{*}{${ }^{130} \mathrm{Xe}$} & $J^{\pi}=0^{+}$ & $J_{\text {samp }}$ & & & & $(n / N)_{k}$ & & \\
\hline & & & & 0.011 & 0.018 & 0.029 & 0.045 & 0.067 \\
\hline & $0_{1}^{+}$ & 0.012 & $E_{k}=$ & -59.141 & -59.273 & -59.369 & -59.435 & -59.481 \\
\hline & $0_{2}^{+}$ & 0.012 & $E_{k}=$ & -57.942 & -58.074 & -58.167 & -58.229 & -58.272 \\
\hline & \multirow[t]{2}{*}{$J^{\pi}=2^{+}$} & \multirow{2}{*}{\multicolumn{2}{|c|}{$J_{\text {samp }}$}} & & & \multicolumn{3}{|l|}{$(n / N)_{k}$} \\
\hline & & & & 0.013 & 0.021 & 0.034 & 0.053 & 0.079 \\
\hline & $2_{1}^{+}$ & 2.002 & $E_{k}=$ & -58.365 & -58.517 & -58.621 & -58.692 & -58.740 \\
\hline & $2_{2}^{+}$ & 2.002 & $E_{k}=$ & -57.699 & -57.866 & -57.994 & -58.082 & -58.144 \\
\hline
\end{tabular}

diagonalized.

In the case of ${ }^{132} \mathrm{Xe}$, the iterative procedure was carried out so as to include up to $40 \% \div 50 \%$ of the basis states. As shown in Fig. 1, about $10 \%$ of the basis states is enough to lead the $J^{\pi}=0^{+}$and $J^{\pi}=2^{+}$eigenvalues to convergence. Indeed, the $J^{\pi}=0^{+}$absolute energies $E_{k}$ decrease by less than $80 \mathrm{keV}$ in going from $n / N \sim 0.08$ to $n / N \sim 0.46$ ( Table 2). The spectrum converges even faster. Indeed, the energy difference $E\left(0_{2}^{+}\right)-E\left(0_{1}^{+}\right)$changes by $\sim 10 \mathrm{keV}$ when we move from $n / N \sim 0.08$ to $n / N \sim 0.46$.

The same convergence rate is found for the levels of ${ }^{130} \mathrm{Xe}$ (Figs. 2 and Table $2)$. Indeed, the fraction of states considered $(7 \% \div 8 \%)$ is enough to bring the energy eigenvalues to the plateau. On the ground of the analysis made for ${ }^{132} \mathrm{Xe}$, the energies obtained here may differ from the asymptotic values $(n / N=1)$ by at most $100 \mathrm{keV}$ or by $10 \div 20 \mathrm{keV}$, when referred to the ground state.

All the corresponding eigenstates have good $J$. As shown in Table 2, even with $7 \% \div$ $8 \%$ of the basis states, the $J$ values coincide with the exact ones up to the second or third 


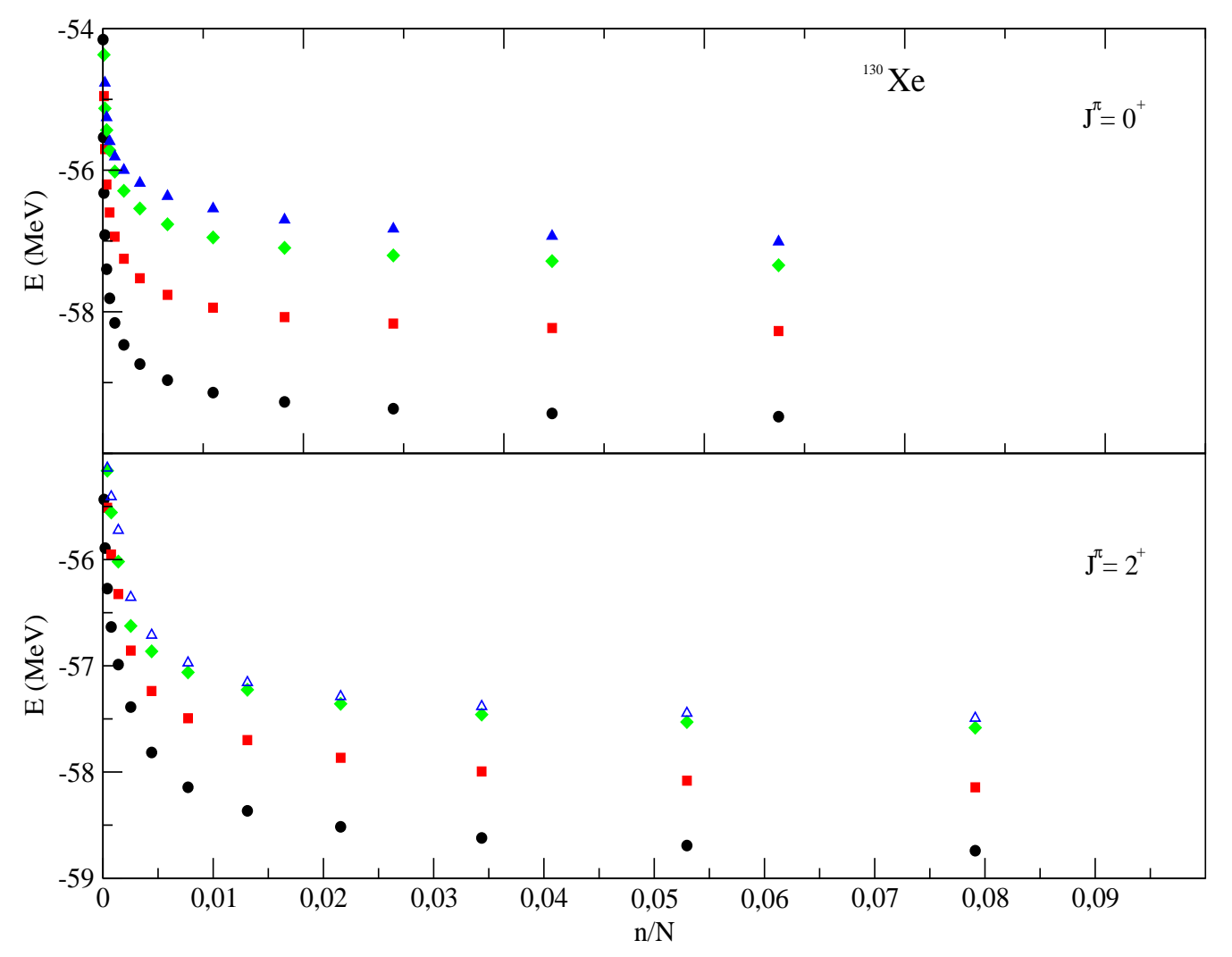

Figure 2. Convergence rate of the lowest $J^{\pi}=0^{+}$and $J^{\pi}=2^{+}$eigenvalues in ${ }^{130} \mathrm{Xe}$.

decimal digit. Thus, although the subspaces selected according to the sampling criterion (9) are not strictly $J$-invariant, the invariance is eventually restored by a relatively small fraction of basis states. Apparently, the components left out by the sampling are small and of little relevance to all observables.

This statement is supported by the good convergence of the $E 2$ transition strengths. In ${ }^{132} \mathrm{Xe}$, the $E 2$ strengths reach their saturated values for $n / N \sim 0.1$. In going from $n / N \sim 0.08$ to $n / N \sim 0.46$, the strengths increases from $B(E 2) \sim 694 \mathrm{e}^{2} \mathrm{fm}^{4}$ to $B(E 2) \sim 708 \mathrm{e}^{2} \mathrm{fm}^{4}$, a $\sim 2 \%$ increment. The same convergence rate is obtained for the $B(E 2)$ values in ${ }^{130} \mathrm{Xe}$. The values obtained for $n / N \sim 0.08$ may be smaller than the asymptotic one by $\sim 2 \% \div 3 \%$.

The good convergence of the angular momentum $J$ and the $B(E 2)$ probes the accuracy of the sampled wavefunctions.

Being interested in illustrating the method, we have refrained ourselves from making any comparison with experiments. We may only say that the computed levels and transition strengths are not far from the corresponding measured quantities [15]. By few small changes, like a better tuning of the single particle energies, the calculation should be able to provide a satisfactory description of the low-energy spectroscopic properties of these two important Xe isotopes. 

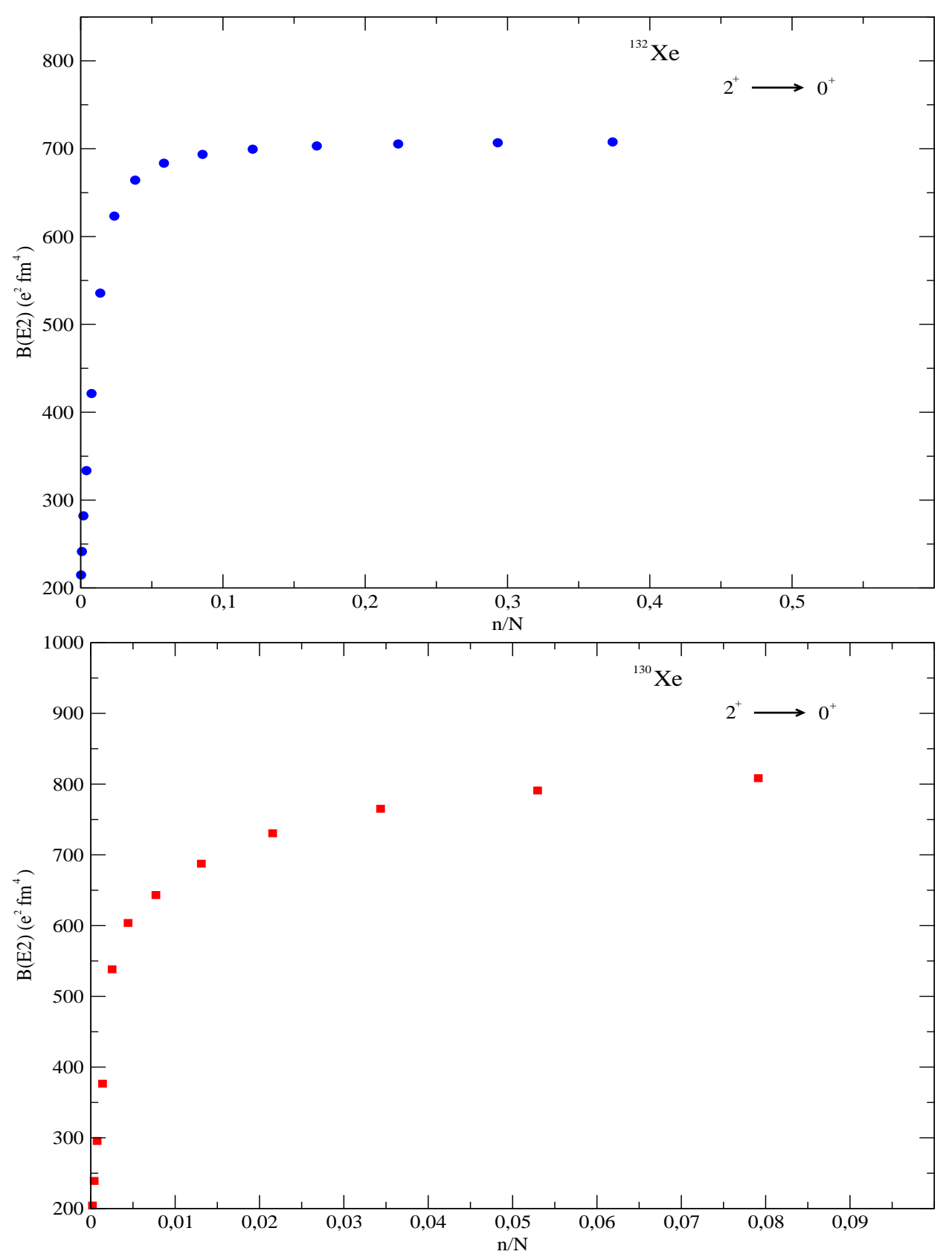

Figure 3. Convergence properties of the $E 2$ reduced strengths.

\section{Conclusions}

From the present analysis we may conclude that the new $m$-scheme implementation of the algorithm allows to take advantage of the sparsity of the Hamiltonian. The importance sampling, combined with sparsity, enhances the convergence of the iterative process leading to an effective truncation of the basis.

The algorithm is able to generate an almost arbitrary number of extremal eigenstates for a given $J^{\pi}$ and, therefore, can describe spectra of great complexity. 
All these eigenstates are automatically orthonormal and have good $J$ values.

Its stability is to be pointed out once again. Indeed, the iterative procedure reaches convergence, independently of the subspace we start with.

As the sizes of the Hamiltonian matrix become too large, the time required to perform the iterations becomes too long, at least on a desktop. On the other hand, the smooth monotonic behavior of energies and transition strengths versus $n / N$ allows to make reliable and straightforward extrapolations to their asymptotic values $(n / N=1)$.

If we stick on a desktop, the extrapolation may allow to treat nuclei, like ${ }^{128} \mathrm{Xe}$, at most. In order to go further, we need to develop a parallel version of the code and explore if and to what extent its performance is enhanced.

\subsection{Acknowledgments}

We thank M. Hjorth-Jensen and N. Pietralla for useful information and stimulating discussions.

\section{References}

[1] Lanczos C 1950 J. Res. Natl. Bur. Stand. 45, 252 (1950)

[2] Golub G H and Van Loan C F 1996 Matrix Computations (Baltimore: John Hopkins University Press)

[3] Caurier E and Nowacki F 1999 Acta Phys. Pol. B 30705

[4] Caurier E, Martnez-Pinedo G, Nowacki F, Poves A and Zuker A P 2005 Rev. Mod. Phys. 77427

[5] Brown B A and Rae W D M 2007 NUSHELL@MSU MSUNSCL Report (unpublished)

[6] Novoselsky A, M. Valliére M and Laadan O 1997 Phys. Rev. Lett. 794341

[7] Koonin S E, Dean D J and Langanke K 1997 Phys. Rep. 2781

[8] Honma M, Mizusaki T and Otsuka T 1995 Phys. Rev. Lett. 751284

[9] Otsuka T, Honma M, Mizusaki T, Shimizu N and Utsuno Y 2001 Prog. Part. Nucl. Phys. 47319

[10] Dukelsky J and Pittel S 2001 Phys. Rev. C 63 R061303

[11] White S R 1993 Phys. Rev. B 4810345

[12] Thakur B, Pittel S and Sandulescu N 2008 Phys.Rev. C 78041303

[13] Andreozzi F, Porrino A and Lo Iudice N 2002 J. Phys. A: Math. Gen. 35 L61

[14] Andreozzi F, Lo Iudice N and Porrino A 2003 J. Phys. : Nucl. Part. Phys. 292319

[15] Coquard L, Pietralla N, Rainovski G, Ahn T, Bettermann L, Carpenter M P, Janssens R V F, Leske J, Lister C. J., Möller O, Rother W, Werner V and Zhu S 2010 Phys. Rev. C 82024317

[16] Hjorth-Jensen M, Kuo T T S and Osnes E 1995 Phys. Rep. 261125

[17] Machleidt R 2001 Phys. Rev. C 63024001 